\begin{tabular}{|l|l|}
\hline $\mathrm{CA}-5$ & 43 \\
\hline
\end{tabular}

$r$
Military Technical College

CAIRO - EGYPT

\title{
A CHEBYSHEV METHOD FOR THE SOLUTION OF BOUNDARY VALUE
}

\section{PROBLEMS}

By

\author{
H.NASR ${ }^{*}$, I. A.HASSANIEN ${ }^{*}$, H.M.EL-HAWAFY ${ }^{*}$
}

\section{ABSTRACT}

An expansion procedure using the Chebyshev polynomials is proposed by using El-Gendi method [1], which yields more accurate results than those computed by D. Hatziavrmidis [2] as indicated from solving the Orr-Somnerfeld equation for both the plane poiseuille flow and the Elasius velocity profile. The present results are also more accurate results than those computed by A.F..Wadia \& F.R.Fayne [3] as indicated from solving the Falkner-Skan equation, which uses a boundary value technique. This method is accomplished by starting with Chebyshev approximation for the highest-order derivative and generating approximations to the lower-order derivatives through integration of the highest-order derivative.

* Military Technical College, Kobbri El-Kobba, Cairo, Egypt. * Faculty of Science, Assiut Unive: sity, Assiut, Egypt. 
Ihird ASAl Conference $4-6$ April 1989), (A) IRI

\section{- INTRODUCTION:}

The use of chebyshev polynomial approximations for the solution of boundary value problem in fluid mechanics has been advocated and developed by orszag [4]. These approximations which include the Galerkin and Tal spectral methods pseudo spectral collocation method, are discussed in detail by Gottlieb and OrEzag [3].

In section II we solve the Orr-Sommerfeld equation. We also show how this method may be applied to solve the Blasius equation which is reduced to a non-1inear algebraic system of equations in the highest derivative and we solve by Newton's method. Comparison of the eigenvalues for either of the plane poiseuille flow or the Blasius velocity profile with thase computed by D.Hatzivarmids [2] are presented.

In section II the problems of laminar or turbulent boundary layer resulting from the flow of an incompressible fluid past a semi-infinite wedge is of considerable practical and theoretical interest. Non-lirear problems with semi-infinite domains are frequently encountered in the study of laminar and turbulent boundar-y layer. Due to the appearance of irregular boundaries, shock waves, boundary layers, derivative boundary conditions, etc., the solutions so obtained have in many cases been unsatisfactory becase of poor resolution, spurious oscillations, and cucessive complter time or storage.

For the past two decades finite difference numerical methods have been used extersively for the evaluation of flow and high speed, viscous and inviscid problem in fluid mechanics. Ferontly the similarity solutions are well studied for the testing finite difference methods. Wadie $\stackrel{0}{ }$ Fayne [b], Ficsenhead [5] ard l. the [7] have been discussed different finite difference schemes for the solutions of the Falkner-Skan equations or Blastis equation. 
In any case the farfield boundary condition is a problem, where the correct value of the unknown shear stress $f$ " at the wall must be faund which ensures an asymptotic appraach of the velocity values $f$, at infinity, to unity, the well known matching condition of viscous solution (near-wall) to the inviscid salutions. Such techniques are termed as "shooting" method. In this paper we introduce a new procedure for the numerical solution based on the Chebyshev approximation using the EL-Gendi method [1] for the numerical solution of the Falkner-..Skan equation. Dur method defines the unknown wall shear stress f', directly without need any carrection interpolation method. We compare our results with values obtained by Wadie \& Fayne [6], Fosenhead [5] and White [7] are shown.

\section{THE ORR-SOMMERFELD EQUATION.}

We choose to apply our method to the solution of the Orr-Sommerfeld equation, which governs the stability of laminar boundary layers in the parallel flow approximation, for plane poiseuille and Elasius boundary layers flows.

Consider the Drr-Sommerfeld equation

$$
\psi^{(4)}-2 a^{2} \psi^{(2)}+a^{4} \psi-i a f\left[(u-\lambda)\left(\psi^{(2)}-a^{2} \psi\right)-u^{(2)} \psi\right]=0,
$$

with

$$
\psi( \pm 1)=\psi^{(1)}( \pm 1)=0
$$

where $\psi(x) \mathrm{e}^{i a(y-\lambda t)}$ is the disturbance stream function in usual normal mode analysis, $u(x)$, is the basic velocity distribution, $F$ is the Fieynolds number based on the boundary layer thickness, $a$ and $\lambda$ are the wave number and wave speed, respectively and super-scripts in parenthesis indicate derivatives with respect to $x$.

To solve equation (1), we put $y^{(4)}(x)=\phi(x)$, then

$$
\psi^{(3)}(x)=\int_{-1}^{x} \phi(x) d x+C_{1} \text {, }
$$


$\psi^{(2)}(x)=\int_{-1}^{x} s_{-1}^{x} \phi(x) \mathrm{d} x \mathrm{~d} x+(x+1) c_{1}+\mathrm{C}_{2}$

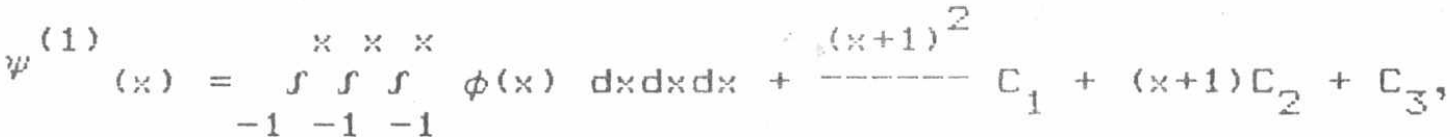

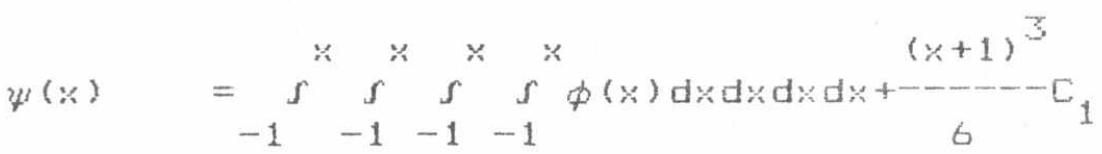

$$
\frac{(x+1)^{2}}{2} C_{2}+(x+1) C_{3}+C_{4}
$$

From the boundary conditions, we get

$$
\begin{aligned}
& c_{3}=c_{4}=0
\end{aligned}
$$

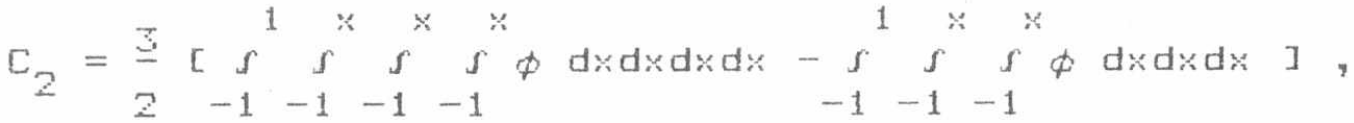

and

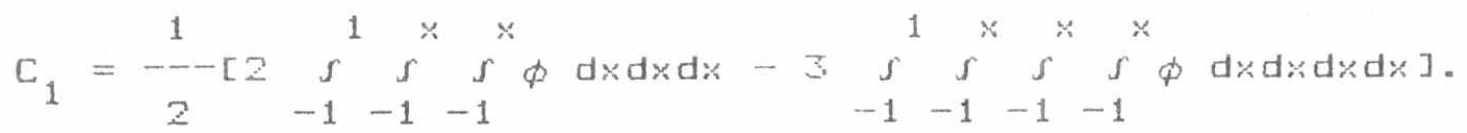

Here, we can give an approximation to equation (2) as follows

$$
\begin{aligned}
& w_{i}^{(3)}=\sum_{j=0}^{N} \ell_{i j}^{(3)} \phi_{j} ., \\
& \psi_{i}^{(2)}=\sum_{j=0}^{N} \ell_{i j}^{(2)} \phi_{j}, \\
& w_{i}^{(1)}=\sum_{j=0}^{N} \ell_{i j}^{(1)} \phi_{j} ., \\
& w_{i}=\sum_{j=0}^{N} \ell_{i j} \phi_{j},
\end{aligned}
$$


where

$$
\begin{aligned}
& \phi_{j}=\phi\left(x_{j}\right), \quad \psi_{i}^{(r)}=\psi^{(r)}\left(x_{i}\right), \quad r=0(1) 4, \\
& x_{i}=-\cos \left(i-\frac{\pi}{N}\right) \quad ; i=O(1) N \text {, } \\
& \ell_{i j}=b_{i j}^{(4)}+\frac{\left(x_{i}+1\right)^{3}}{12}\left(2 b_{N j}^{(3)}-3 b_{N j}^{(4)}\right)+-\frac{3}{4}\left(x_{i}+1\right)^{2}\left(b_{N j}^{(4)}-b_{N j}^{(3)}\right), \\
& \ell_{i j}^{(1)}=b_{i j}^{(3)}+\frac{\left(x_{i}+1\right)^{2}}{4}\left(2 b_{N j}^{(3)}-3 b_{N j}^{(4)}\right)+\frac{3}{2}\left(x_{i}+1\right)\left(b_{N j}^{(4)}-b_{N j}^{(3)}\right), \\
& \ell_{i j}^{(2)}=b_{i j}^{(2)}+\frac{\left(x_{i}+1\right)}{2}\left(2 b_{N j}^{(3)}-3 b_{N j}^{(4)}\right)+\frac{3}{2}\left(b_{N j}^{(4)}-b_{N j}^{(3)}\right), \\
& \ell_{i j}^{(3)}=b_{i j}+\frac{1}{2}\left(2 b_{N j}^{(3)}-3 b_{N j}^{(4)}\right),
\end{aligned}
$$

and

$$
b_{i j}^{(r)}=\frac{\left(x_{i}-x_{j}\right)^{r-1}}{(r-1) !} b_{i j}, r=2(1) 4 \quad, i, j=0(1) \mathrm{N}
$$

where $b_{i j}$ are the elements of the matrix $B$, as mentioned in [1]

Substituting equation (4) in equation (1), we arrive at the following algebraic eigenvalue problem

$$
(A-\lambda D) \phi=0
$$

where $\Phi=\left[\phi_{0} \phi_{1} \cdot \cdots \cdot \phi_{\mathrm{N}}\right]$.

In the equation (5) $A$ and $D$ are $(N+1):(N+1)$ matrices with the elements

$$
a_{m n}=\delta_{m n}-\left(2 a^{2}+i a F u\right) \ell_{m n}^{(2)}+\left(a^{4}+i a^{3} F u+i a F u(Z)\right) \ell_{m n}
$$

and

$$
d_{m n}=-i a F \ell_{m n}^{(2)}+i a^{2} F \ell_{m n}
$$

Numerical solution of the matrix eigenvalue problem (5) is obtained using the QZ method with NAGLIERAFiY routine FOzGJF. 
II.1. Flane Foiseuille Flow

Here $u(x)=1-x^{2}, x \in[-1,1]$. For the plane Foiseuille flow, the eigenfunctions are either symmetric or asymmetric about $x=0$. Then for $N$ even the (N+1) equations in (5) are reduced into the following $(M+1)$ equations where $M=N / 2$.

$$
\left(A^{(1)}-\lambda D^{(1)}, \Phi=0\right.
$$

where

$$
\begin{array}{ll}
a_{i j}^{(1)}=a_{i j}+a_{i(N-j)} & , j=0(1) M-1, \quad i=0(1) M, \\
a_{i M}^{(1)}=a_{i M} & , i=0(1) M, \\
d_{i j}^{(1)}=d_{i j}+d_{i(N-j)}, j=0(1) M-1, \quad i=0(1) M,
\end{array}
$$

and

$$
d_{i M}^{(1)}=d_{i M} \quad, \quad i=0(1) M
$$

In Table 1 we show the mast unstable eigenvalue (which corresponds to a symetric eigenfunction, computed here and in [2] for $a=1$ and $F=10000$ where the exat value is

$$
0.23752649+0.00373967 i \text { [4]. In table } 2 \text { we show the most }
$$
unstable eigenvalue for the critical Fieynolds number and wave number, $F_{i}=5772.22$ and $a_{c}=1.02056$. The values generated by our method compares well with the value obtained by Orszag [4], $0.26400174+5.9 \times 10^{-10} i$.

\section{2. Blasius Velacity Frofile}

The Elasius boundary-layer flow for which the basic flow u is given in terms of a function $f(n)$ which satisfies

$$
\begin{array}{ll}
f^{\prime},+f f^{\prime}=0 & , n \in[0, \infty) \\
f(0)=f^{\prime}(0)=0 \quad \text { and } \quad f^{\prime}(n \rightarrow \infty) \sim 1
\end{array}
$$

where super-scripts denote derivatives with respect to $n$. The infinity condition will be invoked at a finite value of $\eta=\eta_{\infty}$. 
Using the algebraic mapping.

$$
x=2-\frac{n}{n_{\infty}}-1
$$

The unbounded region $[0, \infty)$ is mapped into the finite domain $[-1,1]$ and the problem expressed by equation (7) is transformed into

$$
\begin{aligned}
& f^{\prime \prime}+-\frac{n}{2} \omega_{-} f^{\prime}=0, \\
& f(-1)=f^{\prime}(-1)=0 \quad, \quad f^{\prime}(1)=\frac{n_{\infty}}{2}
\end{aligned}
$$

where superscripts denote derivatives with respect to $\cdots$.

The basic flow for the Blasius problem is obtained as

$$
u(x)=f^{\prime}(x) .
$$

To solve equation ( 9 ), we put $f^{\prime \prime \prime}(k)=\phi(x)$, then by using our method we can give approximations to the function $f(x)$ and its derivatives as follows:

$$
\begin{aligned}
f_{i} & =\sum_{j=0}^{N} \ell_{i j} \phi_{j}+d_{i}, \\
f_{i}^{\prime} & =\sum_{j=0}^{N} \ell_{i j}^{(1)} \phi_{j}+d_{i}^{(1)}, \\
f_{i}^{\prime}= & \sum_{j=0}^{N} \ell_{i j}^{(2)} \phi_{j}+d_{i}^{(2)},
\end{aligned}
$$

where

$$
\phi_{i}=\phi\left(x_{i}\right), f_{i}=f\left(x_{i}\right), f_{i}^{\prime}=f^{\prime}\left(x_{i}\right), f_{i}^{\prime}=f^{\prime}\left(x_{i}\right),
$$

and for $i, j=O(1) N$

$$
\begin{array}{ll}
\ell_{i j}=b_{i j}^{(3)}-\frac{\left(x_{i}+1\right)^{2}}{4} b_{N j}^{(2)}, & d_{i}=\left(x_{i}+1\right)^{2} \frac{n_{\infty}}{B^{-}} \\
\ell_{i j}^{(1)}=b_{i j}^{(2)}+\frac{\left(x_{i}+1\right)}{2} b_{N j}^{(2)}, & d_{i}^{(1)}=\left(x_{i}+1\right) \frac{n_{\infty}}{4},
\end{array}
$$




$$
\ell_{i j}^{(2)}=b_{i j}-\frac{1}{2} b_{i j}^{(2)}, \quad d_{i}^{(Z)}=-\frac{n_{\infty}}{4}
$$

where

$$
b_{i j}^{(3)}=\frac{\left(x_{i}-x_{j}\right)^{2}}{2} b_{i j}, b_{i j}^{(2)}=\left(x_{i}-x_{j}\right) b_{i j}
$$

and $b_{i j}$ are the elements of the matrix $E$ defined in [1].

By using equation (11) equation (9) is transformed into the following system of non-linear equations.

$$
\begin{gathered}
\phi_{i}+\frac{n^{\infty}}{2}-\left(\sum_{j=0}^{N} \ell_{i j} \phi_{j}+d_{i}\right)\left(\sum_{j=0}^{N} \ell_{i j}^{(2)} \phi_{j}+d_{i}^{(2)}\right)=0, \\
i=0(1) \mathrm{N}
\end{gathered}
$$

In Table 3 we show the most unstable eigenvalue for the Blasius flow, obtained by ohir method for $a=0.179$ and $k=580$. For comparison, values obtained by D.Hatziavramidis [2] are shown where the exact value is $0.36412286+0.00795972 \mathrm{i}$ [8]

\section{I I. ME:THOD OF SOLUTYON}

Consider the Falkner-Skan equation for stagnation flows with the similarity property [5, 7].

$$
f^{\prime \prime \prime}(n)+\alpha f(n) f^{\prime \prime}(n)+\beta\left[1-f^{\prime 2}(n)\right]=0
$$

together with the boundary conditions:

$$
f(0)=f^{\prime}(0)=0 \text { and } f^{\prime}(n \rightarrow \infty)=1 \text {. }
$$

Here $\alpha$ is assumed constant, $\beta$ is a measure of the pressure gradient. The prime denotes differentiation with respect to $n$. The special case of the Blasius similarity relation for incompressible viscous flow along $a$ flat plate results when $\beta=1$ and $\beta=0$.

The domain $0 \leq n \leq n_{\infty}$; where $n_{\infty}$ is one end of the user specified computational domain. Using the algebraic mapping 


$$
\therefore=\frac{2 n}{n_{\infty}}-1
$$

The unbounded region $[0, \infty)$ is mapped inta the finite domain $[-1,1]$ and the problem expressed by equation $(13),(14)$ is transformed into

$$
f^{\prime} \cdot+\alpha \frac{n_{\infty}}{2}+f^{\prime} \cdot+\beta\left[\left(\frac{n_{\infty}}{2}, 3^{3}-\left(\frac{n_{\infty}}{2}, f^{\prime} z_{3}\right]\right.\right.
$$

together with the boundary conditions

$$
f(-1)=f^{\prime}(-1)=0 \quad \text { and } \quad f^{\prime}(1)=-\frac{n}{2}
$$

where the prime denotes differentiation with respect to $x$

Our technique is accomplished by starting with Chebyshev approximation for the highest-order derivative, f',', and generating approximation to the lower-order derivatives, f ' ', f' and $f$, through integration of the approximation of the highest-order derivative as follows:

$$
\begin{aligned}
& \text { Setting } \phi(x)=f \cdots(x) \text { then, by integration, we get } \\
& f^{\prime \prime}(x)=s_{-1}^{x} \phi(x) d x+C_{1} \\
& f^{\prime}(x)=s_{-1}^{x} \int_{-1}^{x} \phi(x) d x d x+(x+1) C_{1}+C_{2}
\end{aligned}
$$

From the boundary conditions $(17)$, we get

$$
c_{2}=c_{3}=0
$$

and

$$
c_{1}=-\frac{n}{4}--\frac{1}{2}-s_{-1}^{1} s_{-1}^{x} \phi(x) d x d x
$$

Therefore, we can give an approximation to equation (18) as 
fallows:

$$
\begin{aligned}
& f_{i}=f\left(x_{i}\right)=\sum_{j=0}^{N} \ell_{i j} \phi_{j}+d_{i}, \\
& f_{i}^{\prime}=f \cdot\left(K_{i}\right)=\sum_{j=0}^{N} \ell_{i j}^{(1)} \phi_{j}+d_{i}^{(1)},
\end{aligned}
$$

and

$$
\tilde{f}_{i}^{\prime}=f^{\prime}\left(K_{i}\right)=\sum_{j=0}^{N} \ell_{i j}^{(Z)} \phi_{j}+d_{i}^{(Z)}
$$

for all $\mathrm{i}=0(1) \mathrm{N}$, where

$$
\begin{aligned}
& \ell_{i j}=b_{i j}^{(3)}-\frac{\left(x_{i}+1\right)^{2}}{4}-b_{N j}^{(2)}, \quad d_{i}=\left(x_{i}+1\right)^{2}-\frac{n_{\infty}}{8} \\
& \ell_{i j}^{(1)}=b_{i j}^{(2)}+\frac{\left(x_{i}+1\right)}{2} b_{N j:}^{(2)}, \quad d_{i}^{(1)}=\left(x_{i}+1\right)-\frac{n_{w_{-}}}{4}, \\
& \ell_{i j}^{(2)}=b_{i j}-\frac{1}{2}-b_{i j}^{(2)}, \quad d_{i}^{(2)}=-\frac{n_{\infty}}{4},
\end{aligned}
$$

where

$$
\begin{aligned}
& b_{i j}^{(S)}=\frac{\left(x_{i}-x_{j}\right)^{2}}{2} b_{i j}, \\
& b_{i j}^{(2)}=\left(x_{i}-x_{j}\right) b_{i j}, \quad \quad i, j=0(1) N
\end{aligned}
$$

and $b_{i j}$ are the elements of the matrix $B$, as mentioned in [1].

By using equation (19) the equation (16) transformed to the following system of non-linear equations

$$
\begin{gathered}
\phi_{i}+\alpha\left(-\frac{n_{\infty}}{2}\right)\left(\sum_{j=0}^{N} \ell_{i j} \phi_{j}+d_{i}\right)\left(\sum_{j=0}^{N} \ell_{i j}^{(2)} \phi_{j}+d_{i}^{(2)}\right) \\
+\beta\left[\left(\frac{n_{\infty}-3}{2}-\left(\frac{n_{\infty}}{2}\right)\left(\sum_{j=0}^{N} \ell_{i j}^{(1)} \phi_{j}+d_{i}^{(1)}, 2\right]=0,(20)\right.\right. \\
i=0(1) N
\end{gathered}
$$


in the highest derivative and we solve it by Newton's method.

\section{NUMERICAL RESULTS:}

The method described here-in has been used to calculate the flow for several different combination of $\alpha$ and $\beta$. Table 4 shows comparison of the wall shear stress $f^{\prime \prime}$ for $N=20$ and different $n_{\infty}$. Table 5 illustrates the effect of the degree of approximation for $\alpha=1$ and $\beta=0.5$ (Homann axisymmetric stagnation $f l o w), \alpha=1$ and $\beta=1$ (Hiemenz flow) All values of $f^{\prime}, f^{\prime}$ are in good agreement with those reported by Fosenhead [5] and white [7]. Decreasing the degree of approwimation, the values of the wall shear strests $f^{\prime \prime}$ and the fluid velocity $f^{\prime}$ do not change after fixed degree of approximation. Table [6] lists the value of $f_{w}^{\prime \prime}$, the wall shear stress, for $n_{\infty}=n_{\infty 11}$, and $n_{\infty}=2 \quad n_{\infty 1}$, for two sample cases of Homann and Hiemenz flows and $\alpha=1, \beta=10$ (a flow with a strongly favorable pressure gradient) for a fixed degree of approximation $N=20$.

The computations were carried out on a VME2955, ICLcomputer.

Table (1): The Most Unstable Eigenvalue of Flane Foiseuille Flow for $a=1, F=10000$

\begin{tabular}{|c|c|c|}
\hline$M+1$ & Fresent Method & D. Hatziavramidis \\
\hline 14 & $0.236981433+0.0032181517 i$ & $0.237235137+0.0041940510 i$ \\
\hline 15 & $0.237237495+0.003665072 i$ & $0.237711145+0.0038379230 i$ \\
\hline 16 & $0.237530489+0.0034384176 i$ & $0.237512114+0.0038506654 i$ \\
\hline 17 & $0.237630658+0.0037645283 i$ & $0.237519937+0.0037373238 i$ \\
\hline 18 & $0.237515439+0.0037577974 i$ & $0.237542640+0.0037998328 i$ \\
\hline 19 & $0.237531318+0.0037542197 i$ & $0.237526382+0.0037769869 i$ \\
\hline 20 & $0.237519668+0.0037484922 i$ & $0.237535288+0.0037894188 i$ \\
\hline 21 & $0.237529756+0.0037451062 i$ & $0.237529654+0.0037744669 i$ \\
\hline 22 & $0.237529122+0.0037496205 i$ & $0.237526039+0.0037518324 i$ \\
\hline 23 & $0.2375279013+0.0037493184 i$ & $0.237533259+0.0037893048 i$ \\
\hline 24 & $0.237527620+0.0037486123 i$ & $0.237529593+0.0037705812 i$ \\
\hline 25 & $0.237527969+0.0037485690 i$ & $0.237528078+0.0037631389 i$ \\
\hline 26 & $0.237528070+0.0037487614 i$ & $0.237533319+0.0037892652 i$ \\
\hline 27 & $0.237527987+0.0037480076 \mathrm{i}$ & $0.237536556+0.0038054500 i$ \\
\hline 20 & $0.237527963+0.00374077 .17 i$ & $0.237529579+0.00377060301$ \\
\hline 29 & $0.237527981+0.0037487605 i$ & $0.237538549+0.0038154 .119 i$ \\
\hline
\end{tabular}


Table (2): The most Unstable Eigenvalue for THe Critical Fieynalds. Number $F_{c}=5772.22$ and Wave number $a_{c}=1.02056$.

\begin{tabular}{|c|c|c|}
\hline$M+1$ & Fresent Method & D. Hatzi avramidis [12] \\
\hline 14 & $0.263746649+4.77294095 \times 10^{-5} \mathrm{i}$ & $0.264157871+1.78540477 \times 10^{-4} i$ \\
\hline 15 & $0.264033131+2.25185862 \times 10^{-4} \mathrm{i}$ & $0.263974543+1.90346143 \times 10^{-4} \mathrm{i}$ \\
\hline $\begin{array}{l}16 \\
17\end{array}$ & $0.264054957+4.38697795 \times 10^{-3} \mathrm{i}$ & $0.264010755+8.70870695 \times 10^{-1} \mathrm{i}$. \\
\hline $\begin{array}{l}17 \\
113\end{array}$ & $0.263994745+6.16,6376409 \times 10^{-6} \mathrm{i}$ & $0.2639999880+2.6503281967 \times 10^{-3}{ }^{-1}$ \\
\hline $\begin{array}{l}113 \\
19\end{array}$ & $0.263998427+1.16036057 \times 10^{-3} \mathrm{i}$ & $0.264002880+2.8816,7435 \times 10^{-3} \mathrm{i}$ \\
\hline $\begin{array}{l}19 \\
20\end{array}$ & $0.263999550+1.45006128 \times 10^{-1} 6^{i}$ & $0.264002108+2.56037714 \times 10^{-3} \mathrm{i}$ \\
\hline $\begin{array}{l}20 \\
21\end{array}$ & $0.264004096+5.80368212 \times 10^{-0} i$ & $0.264002649+3.09871639 \times 10^{-5} \mathrm{i}$ \\
\hline $\begin{array}{l}21 \\
22\end{array}$ & $0.264002190+6.66335930 \times 10^{-6}$ & $0.264001857+2.183119503 \times 10^{-6}$ \\
\hline 23 & $\begin{array}{l}0.264001755+6.11743756 \times 10 \\
0.264002024+5.02002144 \times 10^{-6} \frac{1}{1}\end{array}$ & $\begin{array}{l}0.264000685+7.60825237 \times 10 \\
0.264002645+3.11928323 \times 10^{-5} .\end{array}$ \\
\hline 24 & $\begin{array}{l}0.264002024+5.82002 .144 \times 10 \\
0.264002143+5.97293879 \times 10^{-6} \\
\end{array}$ & $0.264001665+1.94065400 \times 10^{-5} \mathrm{i}$ \\
\hline 23 & $0.264002075+6.01441200 \times 10^{-6} \mathrm{i}$ & $0.264001272+1.46951159 \times 10^{-3} \mathrm{i}$ \\
\hline 26 & $0.264002060+5.90590770 \times 10^{-6} i$ & $0.264002646+3.11933774 \times 10^{-5} \mathrm{i}$ \\
\hline 27 & $0.264002073+5.98201964 \times 10^{-6} i$ & $0.264003496+4.14084377 \times 10^{-3} \mathrm{i}$ \\
\hline 28 & $0.264002074+5.98817384 \times 10^{-6} i$ & $0.264001665+1.941 .10240 \times 10^{-3} \mathrm{i}$ \\
\hline 29 & & $0.264004018+4.76961341 \times 10^{-3} i$ \\
\hline
\end{tabular}

Table (3): The Most Unstable Eigenvalue for The Blasius Flow for $a=0.179, \quad F i=580$

\begin{tabular}{|c|c|c|c|c|}
\hline$n_{\infty}$ & $N-1$ & Fresent & Method & D. Hatziavramidis [2] \\
\hline $\begin{array}{l}10 \\
10\end{array}$ & $\begin{array}{l}36 \\
44\end{array}$ & $\begin{array}{l}0.367592246+ \\
0.367588206+\end{array}$ & & $\begin{array}{l}0.367592887+0.00643824879 i \\
0.367595570+0.00644268539 i\end{array}$ \\
\hline $\begin{array}{l}20 \\
20 \\
20\end{array}$ & $\begin{array}{l}36 \\
44 \\
46\end{array}$ & $\begin{array}{l}0.363916305+ \\
0.364106627+ \\
0.364175339+\end{array}$ & $\begin{array}{l}0.00674679702 i \\
0.00784644603 i \\
0.00793035553 i\end{array}$ & $\begin{array}{l}0.363996006+0.00767898122 i \\
0.364154863+0.00797282057 i \\
0.364119456+0.00796321134 i\end{array}$ \\
\hline $\begin{array}{l}30 \\
30 \\
30\end{array}$ & $\begin{array}{l}40 \\
42 \\
44 \\
46\end{array}$ & $\begin{array}{l}0.362303015+ \\
0.364116688+ \\
0.363602568+ \\
0.364215651+\end{array}$ & $\begin{array}{l}0.00315191622 \mathrm{i} \\
0.00641033755 \mathrm{i} \\
0.00690295526 \mathrm{i} \\
0.00663699103 \mathrm{i}\end{array}$ & $\begin{array}{l}0.363403437+0.00908507014 i \\
0.363341298+0.00824699194 i \\
0.363851749+0.0078842921 \\
0.364156290+0.00803473966 i\end{array}$ \\
\hline
\end{tabular}

The computations were carried out on VME 2855, ICL camputer. 
Table (4): Comparison of the Wall Shear Stress f'.'.

\begin{tabular}{|c|c|c|c|c|c|c|c|}
\hline$n_{\infty}$ & $\alpha$ & $\beta$ & Fresent Method & $\begin{array}{l}\text { Wadia } \\
\text { Fayne }\end{array}$ & [2] & $\begin{array}{c}\text { Fosenheac } \\
{[3]}\end{array}$ & $\begin{array}{l}\text { White } \\
\text { [4] }\end{array}$ \\
\hline 8.11 & 1 & -10.15 & -0.1334299 & -0.131999 & & -0.132 & - \\
\hline 8.0 & 1 & -0.18 & $-0.0976,934$ & -0.097 & & -0.0197 & - \\
\hline 5.6 & 1 & -0.14 & 0.1289902 & 0.1285 & & 0.1285 & 0.12864 \\
\hline 5.2 & 1 & -0.15 & 0.2167541 & 0.216101 & & 0.2161 & - \\
\hline 6.9 & 1 & 0.0 & 0.469600 & 0.469604 & & 0.4696 & 0.4696 \\
\hline 4.4 & 1 & 0.3 & 0.774783 & 0.774764 & & - & 0.77476 \\
\hline 3.7 & 1 & 0.5 & 0.927805 & 0.925999 & & 0.928 & - \\
\hline 3.5 & ] & 1 & 1.232617 & 1.23247 & & 1.233 & 1.23259 \\
\hline 3.1 &. $\mathrm{l}$ & 2 & 1.687226 & 1.6866 & & 1.687 & L. 68722 \\
\hline 4.6 & 0 & 1 & $1.1,4712$ & 1.1547 & & 1.1547 & - \\
\hline 3.6 & -1 & 4 & 2.272784 & 2.1602 & & 2.273 & - \\
\hline 10.0 & -1 & 1 & $1.08636,2$ & 1.0628 & & 1.086 & - \\
\hline 2.0 & 1 & 10 & 3.675234 & 3.33 & & - & - \\
\hline 2.11 & 1 & 13 & $4.49148 \%$ & 3.95167 & & - & - \\
\hline 2.0 & 1 & 20 & $\therefore .11301718$ & 4.303 .11 & & - & - \\
\hline
\end{tabular}

Table (5): The Effect of The Degree of Approximation on The Fluid velocity ' $f$ ', $n_{\infty}=4$.

\begin{tabular}{|c|c|c|c|c|c|c|}
\hline$\therefore, \beta$ & $\alpha x$ & $\beta=$ & 0.5 & $\alpha=$ & $\beta=$ & 1 \\
\hline$N$ & 10 & 20 & 40 & 10 & 20 & 40 \\
\hline$n$ & $f^{\prime}$ & $f^{\prime}$ & $f^{\prime}$ & $f^{\prime}$ & $f^{-}$ & $f^{\prime}$ \\
\hline U.U97886 & 0.0888414 & 0.01313416 & 0.0883416 & 0.115869 & $0.11,869$ & 0.115869 \\
\hline 0.381966 & 179: & .317912 & 179 & & 99047 & 0.35 \\
\hline 11.824429 & 0.59624 & $.9626 \%$ & 962 & 98965 & 0.69018563 & 0.698563 \\
\hline 2.111 & 0.19423 & 11.942351 & ia?" & .9732 .29 & $0.17 \% 26$ & 0.973226 \\
\hline 2.618034 & U.906,U1 & 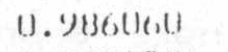 & 0.946150 & แ.ソy4922. & (1.9492! & 1.9\%4925 \\
\hline 3.618034 & 0.999436 & 0.9994218 & 0.999428 & 0.999948 & 0.999849 & 0.999849 \\
\hline 4.000000 & 1.000000 & 1.0000000 & 1.000000 & 1.000000 & 1.000000 & 1.000000 \\
\hline$f_{w}^{\prime \prime}$ & 0.9277187 & $0.92 .77 .1 \% 0$ & 0.927719 & 1.232591 & 1.232591 & 1.232591 \\
\hline
\end{tabular}

Table (6): The Wall Shear Strees f̈'for Different Values of $n_{\infty}$

\begin{tabular}{|c|c|c|c|c|c|c|}
\hline$\alpha$ & 1 & 1 & 1 & 1 & 1 & 1 \\
\hline$\beta$ & 1.0 & 1.0 & 0.5 & 0.5 & 10.0 & 10.0 \\
\hline$n \infty$ & 3.5 & 7.0 & 3.7 & 7.4 & 2.0 & 4.0 \\
\hline
\end{tabular}

Fresent 1.232617 1.232588 $0.92780540 .9276800 \quad 3.675234$ 3.675234 Method

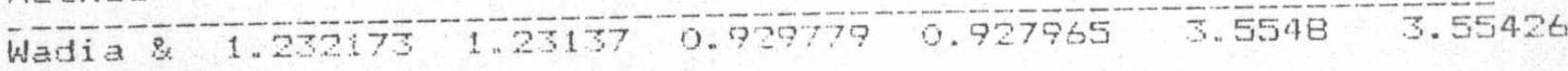
Fayne[z]

Whito[4] 1.232589 0. 


\section{REFERENCES}

[1] El-Gendi, 5.E. (1969): "Chebyshev Solution of Differential, Integral and Integro Differential Equations" Computer J. 12, 282-287.

[2] Hataiavramidis, D.\& Hu, H.C. (1985;: "An Integral Chebyshev Expansion. Method for Ecurdary-value Froblems of 0.D.E. Type", Comp.8 Maths.with App15. 11, 581-586.

[ङ] Orszaq, S.A.8 Gottlieb (1977)" "Numerical Analysis of Spectral Methods: Theory and Applications", SIAM Fral iadelpitia.

[4] Grszag, S.A. (1971): "Accurate Solution of the Orr-Sommerfeld Stability Equation". J.Fluid Mech. 50, $689-703$

[5] Fosenhead, L. (1963): "Laminar Eundary Layers" Oxford University-press

[b] Wadia, A.F. EFayne, F.Fi. (1981): "A Equndary Value Terhnique for the analysis of Laminar Boundary Layer Flows" Intern. J. Computer Math.9. 165-172.

[7] White,F.M. (1974): "Viscous Fluid Flow" McGraw-Hill. Inc.

[e] Zebib, A. (1984): "A Chebystev Method for The Solution of Eoundar" Value Froblems", J. of Comput. Fhysics 5s, 443-455. 